\title{
NON-INVASIVE OPTICAL METHOD FOR EPIDERMAL THICKNESS ESTIMATION
}

\author{
${ }^{1}$ Adrian Peña, ${ }^{2}$ Miguel Arronte, \\ ${ }^{2}$ Eduardo De Posada, ${ }^{3}$ Luis Ponce and ${ }^{3}$ Teresa Flores \\ ${ }^{1}$ Universidad Autonoma de Tamaulipas, Centro Universitario Tampico Madero, CP89109, Mexico \\ ${ }^{2}$ BRALAX S de RL Mi, Ave. Las Torres, N220, Col. Chapultepec, Tampico 89356, Tamps, Mexico \\ ${ }^{3}$ IPN CICATA ALTAMIRA, km 14,5 Carretera Tampico Puerto I, Altamira 89600, Tamps, Mexico
}

Received 2014-03-25; Revised 2014-06-20; Accepted 2014-06-25

\begin{abstract}
A non-invasive method to determine epidermal thickness from the intensity of transmitted visible light is proposed. A simple model for passing light through the epidermis layers, then measuring what is transmitted back out of the surface, is implemented by using the Montecarlo method. Simulations revealed that the transmittance is linearly dependent on the thickness of the epidermis and so demonstrated the viability of the proposed method.
\end{abstract}

Keywords: OCT, Epidermal Thickness, Transmittance

\section{INTRODUCTION}

Thickness of human epidermis is an important topic while studying damage or diseases that are directly related to it or even those that indirectly change some of its characteristics (Huntley, 1989).

Techniques that can be used to measure the thickness of the epidermis can be categorized in two groups: invasive and non-invasive. Invasive techniques require thin cross-sections obtained from biopsies and prepared using the conventional paraffin-formalin preservation method. This technique, which offers a greater amount of information about the sample, has less than $1 \mu \mathrm{m}$ resolution in deep tissues. Unfortunately, during preparation of the tissue, it deforms and changes the thickness of the sample,reason why this technique is not recommended for exact epidermal thickness measurements.

Non-invasive techniques (Meurer et al., 2004), such as LSM "laser scanning microscopy" and OCT "optical coherence tomography", had been used to determine epidermal thickness. The latter has a typical resolution of $10-30 \mu \mathrm{m}$. It can be used to obtain images at depths in the range of millimeters, when a contrasting agent is applied. On the other hand, LSM is considered a highresolution technique with less than $1 \mu \mathrm{m}$ resolution. It has the disadvantage that a fluorescent agent must be injected to ensure a good image and to determine the exact thickness of the epidermis. LSM technique yields very good quality horizontal images of the skin, whereas vertical, or in-depth, images are better captured using OCT. Clearly, OCT and LSM can be efficiently used to determine skin thickness, but these techniques are expensive and difficult to implement. Lademann et al. (2007) carried out a comparison between OCT, LSM and the histological technique. They showed that the three techniques may be used to obtain information on the structure of skin layers with similar results. Recently, infrared diffuse reflection spectroscopy has emerged as a new technique to determine epidermis and dermis thickness (Miyamae et al., 2012). Its major advantage relies on its simplicity to implement and its accuracy. We propose an alternative to the above mentioned technique in the present study: a simple and economic technique, which is non-invasive and capable of determining epidermal thickness as a function of the measured transmittance in the visible region of the spectrum. Corresponding Author: Adrian Peña, Universidad Autonoma de Tamaulipas, Centro Universitario Tampico Madero, CP89109, Mexico. 


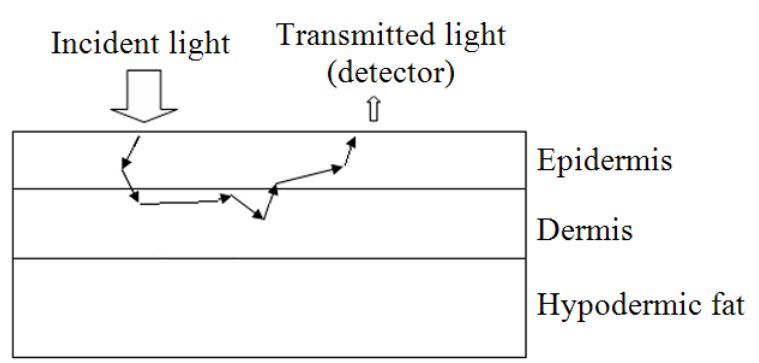

Fig. 1. Drawing of a hypothetical trajectory of a scattered single photon within the skin.

The proposed method is based on the study made by Meglinski and Matcher (2001) in which the spatial distribution of detector sensitivity, inside a multilayer medium with strong scattering and absorption, is analyzed. Figure 1 shows a schematic diagram of a multilayered structure with thickness and optical properties similar to those of the epidermis, dermis and a section of hypodermic fat. In addition, it is schematically shown how the transmitted signal is measured; also path randomness presented by photons in skin is observed.

\section{MATERIALS AND METHODS}

\subsection{Experimental Setup}

As seen in Figure 2, the experimental setup consists of a halogen light source (Mod.LS-1-LL Ocean Optics), an Ocean Optics spectrometer (USB4000) and a reflectance fiber (R200-7-VIS/NIR). The reflectance fiber has a central fiber and six outer fibers joined together. Each of the fibers has a diameter of $200 \mu \mathrm{m}$ and they are embedded in a stainless steel cap that is $3.0 \times 0.25$ inches. The central fiber is connected to the spectrometer and the outer fibers are connected to the light source, such that one collection fiber and six illumination fibers are available. The light emitted from the source travels across all of the outer fibers until it reaches the skin. A portion of the reflected beam is collected by the central fiber, which carries the beam to the spectrometer. For practical purposes, we consider the epidermis to be a single structure.

\subsection{General Procedure}

Experimental measurements of transmittance intensity in the center of the tactile elevations in each fingertip of the right hand were done with an initial population of 10 persons. All subjects were adult volunteers and no ethical issues were involved during the experiment. The acquired spectra were analyzed twice at $632 \pm 2 \mathrm{~nm}$. Means and standard deviations were calculated for each acquired spectrum. Figure 3 shows the mean transmittance of five fingers in one element of the sample.

In order to calibrate our measurements, we determined the thickness of the fingertips using OCT images and then related them to the transmittance intensity measured for each fingertip. As described by Meglinksi et al. (2001), the spatial distribution of the detector can give information that the major contribution of the detected signal is produced by upper layers. However, as the detector distance increases, the contribution of the deeper layers is significant as well. Based on this, we established that the major contribution of the transmitted light is from the epidermis.

OCT images were acquired at the same spot from which the spectra was determined. The images were all collected using a commercial OCT (Thorlabs SR-OCT 930). This instrument has a central wavelength of $930 \mathrm{~nm}$, maximum image depth of $1.6 \mathrm{~mm}$ and an axial resolution of $6.2 \mu \mathrm{m}$. A typical OCT image of a thick thumb can be seen in Figure 4. Once the images were acquired, it is identified the epidermal-dermal boundary by an imaginary line. For each image, ten data points (over the line mentioned above) were chosen to obtain the median epidermal thickness. Figure 5 shows the epidermal thickness measurements for thumb, ring, median, index and little finger for one subject from the sample.

Figure 6 shows the mean intensity of the transmittance measured in vivo, as a function of the mean epidermal thickness (obtained by OCT images). It is noted that intensity, as a function of epidermal thickness, can be fit to a straight line, so the thickness of the epidermis can be predicted for each measured transmittance value.

Following the methodology described above, Figure 7 shows how the transmittance intensity varies as a function of epidermal thickness. Five different subjects with different skin colors are represented in the graph. Each subject is displayed in a different color and each finger is represented with numbers from 1 to 5 . A percentage of the transmitted light is absorbed due to the content of melanin in the epidermis (Zonios et al., 2008). Human skin colors are primarily due the presence of melanin in the skin (Alaluf et al., 2002). It can be seen from Figure 7 that the percentage of the transmitted light linearly decreases due to increments of the epidermal thickness. Melanin content in epidermis of different subjects affects the slope of transmittance as a function of the epidermal thickness. 


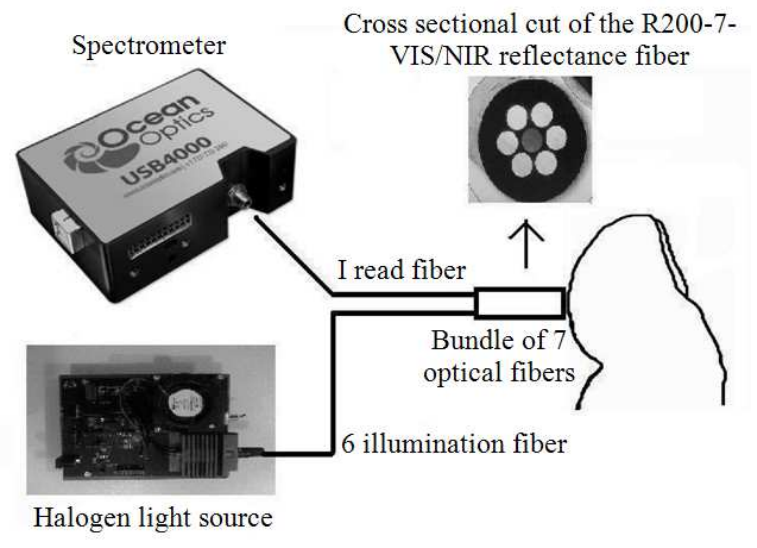

Fig. 2. Schematic description of the experimental setup used to acquire the transmitted intensity

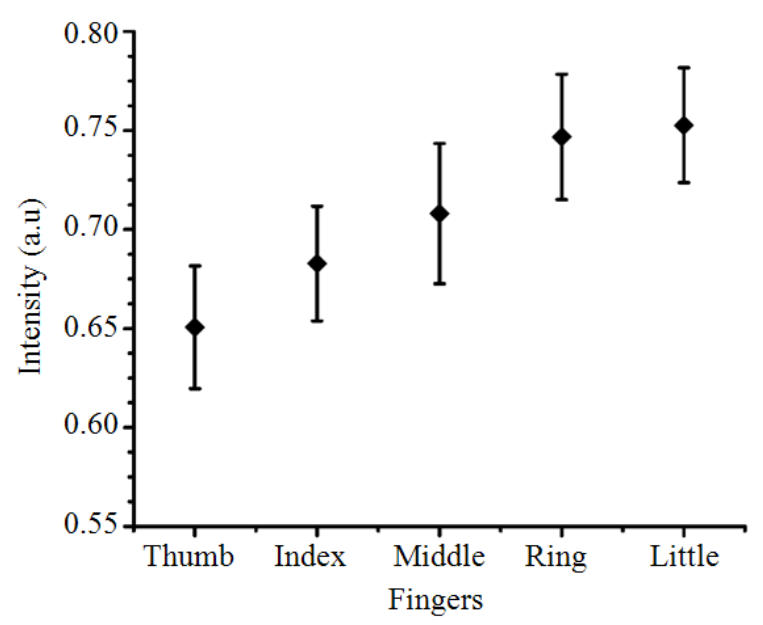

Fig. 3. Intensity measurements using five fingers of a subject from the sample. Error bars: Standard deviation

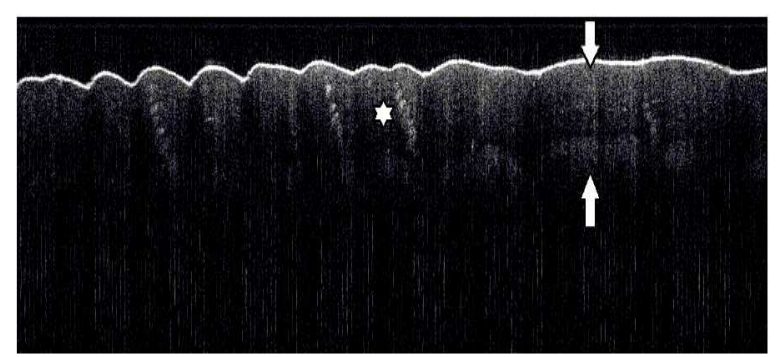

Fig. 4. Cross-section OCT image in the center of the tactile elevation of a thick thumb. The border between stratum cornea and epidermis can be seen, whereas epidermal and dermal border is less clear. The six-point star shows sweat glands in the stratum corneum. OCT image: $2 \times 0.8 \mathrm{~mm}$

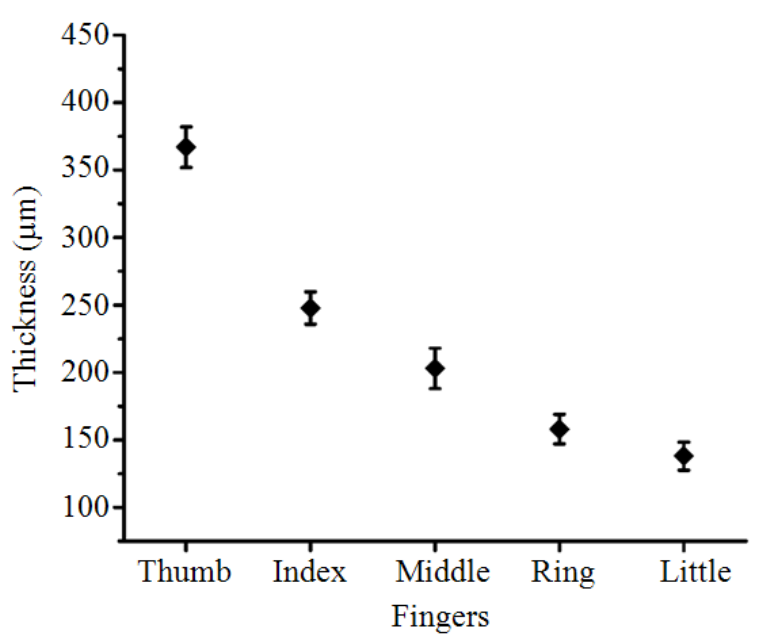

Fig. 5. Epidermal thickness measurements obtained by OCT images. Error bars: Standard deviation

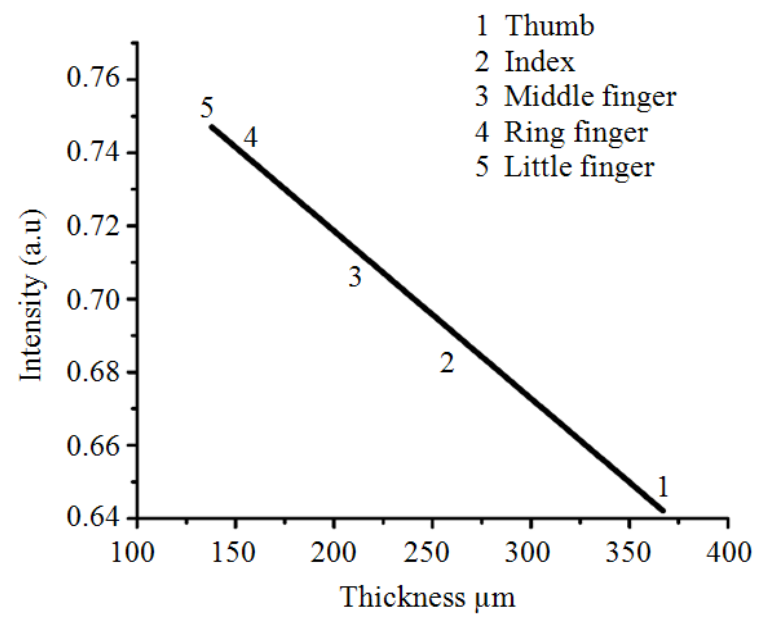

Fig. 6. Transmittance measured in vivo as a function of epidermal thickness obtained by OCT

\section{DISCUSSION}

In previous studies, different techniques used to predict the thickness of epidermis were analyzed (Huntley, 1989) (histological cross sections cuts, OCT and LSM). Each of these techniques has characteristics that make it suitable for specific applications. This study demonstrates that a noninvasive technique, using transmittance as a measurement factor, can indeed determine epidermal thickness. 


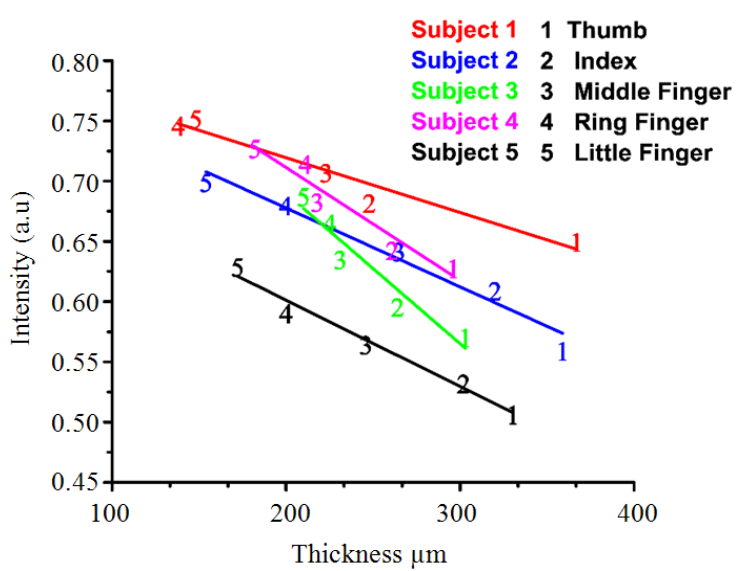

Fig. 7. Transmittance measured in vivo as a function of epidermal thickness in five subjects of the sample

The experimental results indicate that the percentage of transmitted light linearly decreases due to increments of epidermal thickness. Figure 7 shows the relationship between the measured transmittance and fingers' epidermal thickness, on five individuals from the sample. It is noted that the thumb has the greatest epidermal thickness and little finger has the least, as described in studies reported by (Fruhstorfer et al., 2000).

In summary, this study confirms that the relationship between the thickness of the epidermis and transmittance can be used as a simple, economic and non-invasive method to measure epidermal thickness. It is shown that by fitting the data to a linear shape, the thickness can be predicted from each transmittance value measured.

\section{CONCLUSION}

The proposed method can effectively estimate thickness variations of the epidermis, by a simple noninvasive transmittance measurement. We found that the content of melanin in the epidermis changes the slope of transmittance as function of epidermal thickness. The proposed technique relies on OCT to associate the thickness to the measured transmittance. It was demonstrated that, once the calibration is done, we are able to predict thickness of the epidermis.

This technique is not intended as a substitute for high accuracy techniques available nowadays. We anticipate that it can be used as a very cheap alternative to determine thickness variations of the epidermis.

\section{ACKNOWLEDGEMENT}

This study was supported by Fondo Mixto CONACYT - Gobierno del Estado de Tamaulipas.

\section{REFERENCES}

Alaluf, S., D. Atkins, K. Barrett, M. Blount and N. Carter et al., 2002. Ethnic variation in melanin content and composition in photo exposed and photo protected human skin. Pigment Cell Res., 15: 112118. DOI: 10.1034/j.1600-0749.2002.10071.x

Fruhstorfer, H., U. Abel, C.D. Garthe and A. Knüttel, 2000. Thickness of the stratum corneum of the volar fingertips. Clin. Anatomy, 13: 429-433. DOI: 10.1002/1098-2353(2000)13:6<429AIDCA6>3.0.CO, $2-5$

Huntley, A.C., 1989. Cutaneous manifestations of diabetes mellitus. Dermatol. Clin., 33: 605-617. DOI: 10.1111/j.1365-4362.1994.tb02915.x

Lademann, J., N. Otberg, H. Richter, L. Meyer and H. Audring et al., 2007. Application of optical noninvasive methods in skin physiology: A comparison of laser scanning microscopy and optical coherent tomography with histological analysis. Skin Res. Technol., $\quad 13 . \quad$ DOI: $10.1111 / \mathrm{j} .1600$ 0846.2007.00208.x

Miyamae, Y., M. Kawabata, Y. Yamakawa, J. Tsuchiya and Y. Ozaki, 2012. Non-invasive estimation of skin thickness by near infrared diffuse reflection spectroscopy-separate determination of epidermis and dermis thickness. J. Near Infrared Spectroscopy, 20: 617-622. DOI: 10.1255/jnirs.1024

Meglinski, I.V. and S.D. Matcher, 2001. Analysis of the spatial distribution of detector sensitivity in a multilayer randomly inhomogeneous medium with strong light scattering and absorption by The Monte Carlo method. Opt. Spectroscopy, 91: 654-659. DOI: $10.1134 / 1.1412689$

Meurer, M., M. Stumvoll and R.M. Szeimies, 2004. Skin changes in diabetes mellitus. Hautarzt, 55: 428-35. PMID: 15083279

Zonios, G., A. Dimou, I. Bassukas, D. Galaris and Tsolakidis et al., 2008. Melanin absorption spectroscopy: New method for noninvasive skin investigation and melanoma detection. J. Bio. Opt., 13: 1-1. DOI: 10.1117/1.2844710 